\title{
A Kuwaiti risk score for detecting diabetes
}

\section{Abdulwahab Shamsaldin, Abdullah Alkandari, Joseph Longenecker, Ahmad Alkhatib, Rihab Al-Wotayan,}

Noël C Barengo, Qais Al Duwairi and Jaakko Tuomilehto

Dasman Diabetes Institute, Kuwait

\section{INTRODUCTION}

- The Middle East and North Africa (MENA) have been heavily hit by the global diabetes epidemic.

- Early detection and cost-effective screening are important health tools in managing and reducing the prevalence of diabetes.

- A national survey of adults was conducted in Kuwait in 2014 to determine the prevalence of non-communicable diseases and their risk factors. Here, we describe a diabetes risk score for a Kuwaiti population.

\section{METHODS}

- 3915 Kuwaiti men and women aged 18-69 were surveyed using the WHO Instrument for Chronic Disease Risk Factor Surveillance

- Three consecutive steps:

I. Demographics and medical history

II. Physical measurements

III. Blood biochemistry, including fasting plasma glucose and HbA1c

- Predictive models of diabetes risk factors were created using logistic regression on IBM SPSS Statistics v.24

\begin{tabular}{|c|c|c|c|}
\hline \multicolumn{4}{|c|}{ RESULTS } \\
\hline \multicolumn{4}{|c|}{ Table 1: Participant characteristics } \\
\hline & Total & Male & Female \\
\hline $\mathbf{n}$ & $3915(100)$ & $1456(37.2)$ & $2459(62.8)$ \\
\hline Age (Years) & $37.2(12.3)$ & $36.9(12.6)$ & $37.3(12)$ \\
\hline BP (Systolic) & $121.8(16.4)$ & $126.9(16.2)$ & $118.6(15.7)$ \\
\hline BP (Diastolic) & $78.2(10.5)$ & $80.9(10.5)$ & $76.6(10.2)$ \\
\hline Height $(\mathrm{cm})$ & $163.7(9.2)$ & $171.9(7.25)$ & $158.6(6.05)$ \\
\hline Weight (kg) & $79.5(17.7)$ & $86.2(17.1)$ & $75.4(16.7)$ \\
\hline BMI $\left(\mathrm{kg} / \mathrm{m}^{2}\right)$ & $29.6(6.15)$ & $29.2(5.62)$ & $29.9(6.44)$ \\
\hline Waist Circumference $(\mathrm{cm})$ & $90.7(15.2)$ & $94.2(15.2)$ & $88.6(14.8)$ \\
\hline Hip Circumference $(\mathrm{cm})$ & $106.6(14.6)$ & $105.8(14.3)$ & $107.1(14.8)$ \\
\hline Total Cholesterol (mmol/l) & $5.05(0.99)$ & $5.09(1.04)$ & $5.02(0.96)$ \\
\hline Triglycerides (mmol/l) & $1.47(1.03)$ & $1.73(1.35)$ & $1.32(0.73)$ \\
\hline HDL Cholesterol (mmol/l) & $1.29(0.31)$ & $1.13(0.27)$ & $1.38(0.3)$ \\
\hline LDL Cholesterol & $3.1(0.86)$ & $3.2(0.91)$ & $3.05(0.81)$ \\
\hline Fasting Plasma Glucose (mmol/l) & $5.74(2.43)$ & $5.9(2.47)$ & $5.65(2.39)$ \\
\hline HbA1c (\%) & $5.81(1.36)$ & $5.83(1.34)$ & $5.80(1.37)$ \\
\hline
\end{tabular}

\section{RESULTS}

Table 2: Logistic regression models with diabetes status as the dependent variable

\begin{tabular}{|c|c|c|c|c|c|}
\hline & \multicolumn{3}{|c|}{ Full model $(n=1842)$} & \multicolumn{2}{|c|}{ Concise model $(n=2064)$} \\
\hline & Beta C. & $\mathrm{OR}(\mathrm{Cl})$ & Sig. & Beta C. & $\mathrm{OR}(\mathrm{Cl})$ \\
\hline Intercept & -3.569 & & & -3.268 & \\
\hline $\begin{array}{c}\text { Are you over } 45 \text { years of } \\
\text { age? }\end{array}$ & 0.84 & $\begin{array}{c}2.32 \\
(1.62-3.34)\end{array}$ & 0.000 & 0.96 & $\begin{array}{c}2.6 \\
(1.87-3.62)\end{array}$ \\
\hline $\begin{array}{l}\text { Have you previously been } \\
\text { diagnosed with diabetes? }\end{array}$ & 1.37 & $\begin{array}{c}3.94 \\
(1.83-8.48)\end{array}$ & 0.000 & 1.10 & $\begin{array}{c}3 \\
(1.47-6.13)\end{array}$ \\
\hline $\begin{array}{l}\text { Are you obese (BMI } \\
\left.\geq 25 \mathrm{~kg} / \mathrm{m}^{2}\right) ?\end{array}$ & 0.42 & $\begin{array}{c}1.53 \\
(1.09-2.14)\end{array}$ & 0.014 & 0.43 & $\begin{array}{c}1.54 \\
(1.12-2.11)\end{array}$ \\
\hline $\begin{array}{c}\text { Is your waist/hip ratio } \\
\geq 0.9 \text { for men or } \geq 0.85 \\
\text { women? }\end{array}$ & 0.58 & $\begin{array}{c}1.79 \\
(1.27-2.53)\end{array}$ & 0.001 & 0.61 & $\begin{array}{c}1.84 \\
(1.33-2.55)\end{array}$ \\
\hline $\begin{array}{l}\text { Are you currently a } \\
\text { smoker? }\end{array}$ & 0.57 & $\begin{array}{c}1.77 \\
(1.16-2.72)\end{array}$ & 0.008 & 0.63 & $\begin{array}{c}1.87 \\
(1.31-2.68)\end{array}$ \\
\hline Are you male? & 0.18 & $\begin{array}{c}1.2 \\
(0.82-1.75)\end{array}$ & 0.353 & & \\
\hline $\begin{array}{l}\text { Do you eat fewer than } 5 \\
\text { portions of fruit or } \\
\text { vegetables a day? }\end{array}$ & 0.13 & $\begin{array}{c}1.14 \\
(0.82-1.6)\end{array}$ & 0.436 & & \\
\hline $\begin{array}{c}\text { Do you exercise (walk or } \\
\text { bike) for less than } 10 \\
\text { minutes a day? }\end{array}$ & 0.30 & $\begin{array}{c}1.34 \\
(0.93-1.94)\end{array}$ & 0.114 & & \\
\hline $\begin{array}{l}\text { Have you previously been } \\
\text { diagnosed with high blood } \\
\text { pressure? }\end{array}$ & -0.26 & $\begin{array}{c}0.77 \\
(0.41-1.47)\end{array}$ & 0.434 & & \\
\hline $\begin{array}{l}\text { Are you taking any } \\
\text { medication for high blood } \\
\text { pressure? }\end{array}$ & 0.46 & $\begin{array}{c}1.57 \\
(0.79-3.13)\end{array}$ & 0.193 & & \\
\hline
\end{tabular}

Figure 1: ROC Curve and optimum cutoff points ROC

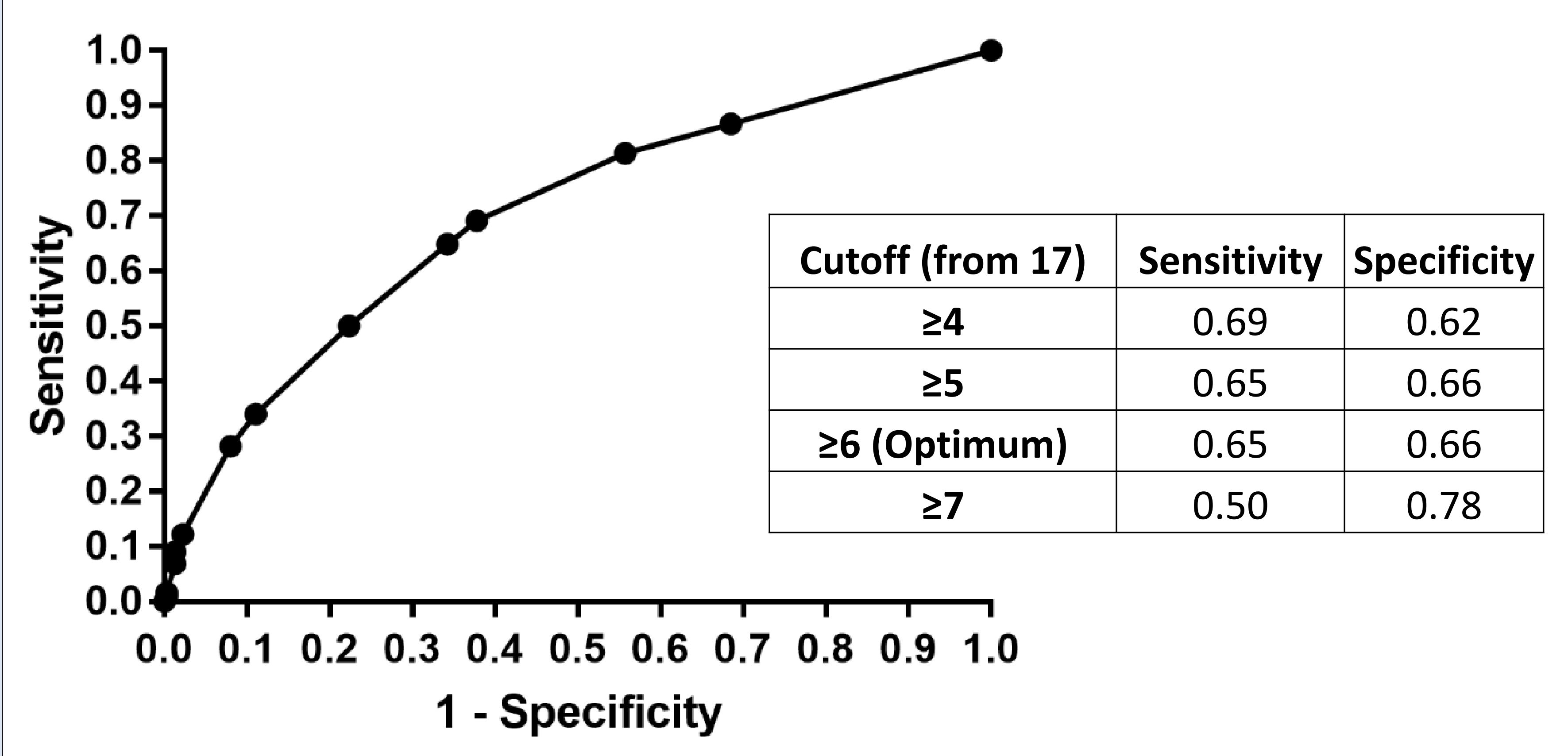

Table 3: The diabetes risk score for the Kuwaiti population

$\begin{array}{ccc}\text { Question } & \text { Yes } & \text { No } \\ \text { Are you over } 45 \text { years of age? } & 4 & 0\end{array}$

Have you previously been diagnosed with diabetes?

\begin{tabular}{|c|c|}
\hline & \\
\hline Are you obese $\left(\mathrm{BMI} \geq 25 \mathrm{~kg} / \mathrm{m}^{2}\right)$ ? & \\
\hline
\end{tabular}

Is your waist/hip ratio $\geq 0.9$ for men or $\geq 0.85$ for women?

3

0

Are you currently a smoker?

3

0

\section{CONCLUSION}

A screening tool capable of detecting diabetes in over two thirds of Kuwaiti adults was created 